ARTICLE

Received 14 Aug 2013 | Accepted 29 Nov 2013 | Published 10 Jan $2014 \quad$ DOl: 10.1038/ncomms4047

\title{
Eye position information is used to compensate the consequences of ocular torsion on $\mathrm{V} 1$ receptive fields
}

\author{
N. Daddaoua ${ }^{1, \dagger}$, P.W. Dicke $^{1} \&$ P. Thier ${ }^{1}$
}

It is commonly held that the receptive fields (RFs) of neurons in primary visual cortex (V1) are fixed relative to the retina. Hence, V1 should be unable to distinguish between retinal image shifts due to object motion and image shifts resulting from ego motion. Here we show that, in contrast to this belief, a particular class of neurons in V1 of non-human primates have RFs that are actually head centred, despite intervening eye movements. They use eye position information to shift their RFs location and to change their orientation tuning on the retina so as to fully compensate for the retinal consequences of a particular type of reflexive eye movements, ocular counter-roll, an eye rotation around the line of sight partially counterpoising head tilt. In other words, V1 uses eye position information to resolve the ambiguity if retinal image tilt is the result of the tilting of an object or of the ocular counter-roll.

\footnotetext{
${ }^{1}$ Hertie-Institute for Clinical Brain Research, Department of Cognitive Neurology, University of Tübingen, 72070 Tübingen, Germany. ${ }^{\dagger}$ Present address: Columbia University, 1051 Riverside Drive Unit 87, Center for Neurobiology and Behavior, Kolb Research Annex, New York, New York 10032, USA.

Correspondence and requests for materials should be addressed to N.D. (email: nd2425@columbia.edu) or to P.T. (email: thier@uni-tuebingen.de).
} 
W e reliably perceive the visual ${ }^{1}$ world to be upright, although tilting the head relative to the gravitational vector may cause substantial tilting of the retinal image. Perceptual invariance to head tilt requires our brain to use information about head tilt and its consequences to reconstruct a world-centred representation of visual objects ${ }^{2-5}$. The orientation of the retinal image is the result of head tilt and ocular counterroll (OC), the latter being a compensatory rotation of the eyes around the line of sight, which mitigates the tilt-induced rotation of the retinal image ${ }^{6-9}$. As yet the neural mechanism underlying the reconstruction of a tilt-invariant representation of the world has remained unknown to us ${ }^{10}$. Nevertheless, it is commonly held that primary visual cortex (V1) is not part of it, and that V1 is unable to distinguish between image rotation due to object-tilt in the world and image rotation resulting from head tilt ${ }^{1}$. Yet, this assumption has never been critically tested.

To test this assumption, we map V1 neurons receptive fields (RFs) before and after inducing OC by tilting the head. We find that V1 neurons sensitive to retinal slip induced by OC are able to shift their RFs on the retina and moreover to change their orientation/direction preferences such as to fully compensate for the visual consequences of OC. This finding suggests that V1 uses non-retinal information on eye position to compensate the visual consequences of eye torsion due to ocular counter-roll.

\section{Results}

Effects of head tilt and OC on RF location. To reveal the role of V1 in dealing with the visual consequences of tilting head and body as a whole, we compared the preferences of V1 neurons of two rhesus monkeys fixating a small dot at $50 \mathrm{~cm}$ distance on a monitor in front of them, either upright or, alternatively, with their head and body tilted as a whole passively in the roll plane. We first determined the RF map of a neuron as well as its orientation/direction preferences with the head and body upright (Fig. 1b lower panel). Then we tilted the animal $50^{\circ}$ about the naso-occipital axis ( = roll tilt) and repeated the measurements. Finally, the animal was rotated back to upright to control the preferences for this head orientation. The direction of the tiltclockwise (cw, Fig. 1c upper panel) versus counterclockwise (ccw, Fig. 1a upper panel)-was pseudorandomized. If possible, we finally repeated the measurements for the head and body tilt orientation opposite to the one tested at the beginning. Fixation was controlled using $2-\mathrm{D}$ search coils ${ }^{11}$, while the amount of tiltinduced $\mathrm{OC}^{12}$, typically around $10 \%$ of the amount of the head tilt, was determined by comparing photographs of the eyes while fixating the fixation target before and after the head tilt. The RF mapping procedures and the assessment of orientation preferences started after the eyes had stabilized in their new position relative to the head, which was the case in less than $10 \mathrm{~s}$ after the head tilt offset. To be able to reveal even subtle influences of tilting on the preferences of neurons, only wellisolated individual neurons were considered for the subsequent analysis. This report focuses on 70 neurons located in layers 2 and 3 according to the criteria of Snodderly and Gur ${ }^{13}$. The validity of the layer assignment is supported by the longer response latencies of neurons in layers 2 and 3 as compared with those in layer 4 (Supplementary Fig. 1).

As mentioned earlier, the common belief is that the RFs of V1 neurons are fixed relative to the retina. Hence, if we map a headfixed location in the visual field (green dotted square in Fig. 1b lower panel) to delineate the RF map of a V1 neuron (black and red dotted circle in Fig. 1b) with the head upright, then after tilting the monkey $50^{\circ} \mathrm{cw}$ (Fig. 1c upper panel) and inducing a $\mathrm{ccw}$ OC around the line of sight, and remapping the same headcentred location (Fig. 1c lower panel), we should expect a rotation of the previously acquired RF map around the line of sight (rotation of the dotted black circle around the line of sight to reach the location of the blue circle in Fig. 1c lower panel). The magnitude and the direction of such a rotation should correspond to the magnitude and direction ( $\mathrm{ccw}$ in this case) of tilt-induced OC.

In contrast to this belief, we recorded neurons whose RFs did not rotate after head roll tilt, thus preserving the same headcentred location and therefore compensating for the tilt-induced OC. Figure 2 compares the RF plots of two exemplary neurons from V1 for the head upright and the head tilted $50^{\circ} \mathrm{cW}$ as established by reverse correlation ${ }^{14}$. The two neurons represent the two qualitatively different patterns of reaction to head tilt. The neuron presented in Fig. 2a had a RF with coordinates that remained fixed relative to the head also after the tilt, thereby fully compensating for the tilt-induced $\mathrm{OC}$ of $3.83^{\circ}$. On the other hand, the neuron shown in Fig. $2 \mathrm{~b}$ sported a RF that preserved its location on the retina, independent of the orientation of the head and independent of head tilt-induced OC.

Further examples of head-centred (Fig. 3d) and retina-centred neurons (Fig. 3b) are depicted in Fig. 3 that also shows their orientation tuning curves as obtained by probing the neurons with optimal (spatial frequency typically between 0.5 and 4.4 cycles per degree, temporal frequency between 1.6 and 5.2 cycles $^{-1}$ ) drifting sinusoidal gratings of different orientation. In many cases, RF after tilts were neither head centred nor retina centred but stayed at varying positions in between the two frames of reference (FOR) (see Fig. 3c for an example). As discussed later, the orientation preference turned out to be a predictor of the FOR that the neuron acquired after the intervening tilt-induced OC.

Figure 4a summarizes the amount of tilt-induced angular RFs rotation around the line of sight (referred to as 'Angular RF shift') of all layer 2 and 3 neurons $(n=70)$ relative to a head-centred FOR for head tilts of $50^{\circ} \mathrm{cw}$ (black symbols on the right side of the panel denote significant angular RF shifts, magenta symbols denote nonsignificant angular RF shifts) and ccw and back to upright. Independent of the direction of the tilt $\left(50^{\circ} \mathrm{cW}\right.$ or $\left.\mathrm{ccw}\right)$ and the RF location (Supplementary Fig. 2), angular shifts of RFs lay within a range from $0^{\circ}$ (head-centred RF) to $4-6^{\circ}$ relative to the head (retina-centred RF), the latter range corresponding to the amount of tilt-induced OC observed (the blue symbols denote OC of the eye contralateral to the downed ear, red symbols the one ipsilateral; left eye OC reconstructed, see Methods). Note that in any case, whenever it was possible to retest the neuron, the RF returned to its original position after the return from the tilt (black and magenta symbols in the middle of the panel). Moreover, as shown in Fig. 5, independent of the varying size of the angular shift, the eccentricity of RFs did not change. This is what one would expect to result from an eye rotation around the line of sight (refer to the Supplementary Information including Supplementary Figs 1 and 3 for further information establishing the validity of the procedures used to delineate RFs and their changes due to head tilts and to Supplementary Fig. 4 and Supplementary Table 1 for a comparison of fixation precision for the three body orientation tested for the example neurons shown in Fig. 3).

Neurons sensitive to OC-induced retinal slip are head fixed. A consideration of the orientation preference of neurons suggests that the tilt-induced RF shifts follow a clear geometrical rule, rather than being arbitrary. Neurons that were orientation selective and retina centred (see Fig. $3 \mathrm{~b}$ for an example; see also Fig. $4 \mathrm{~d}$ right side for illustration. In this illustration the angles exaggerate the actual measurements for the sake of clarity) 


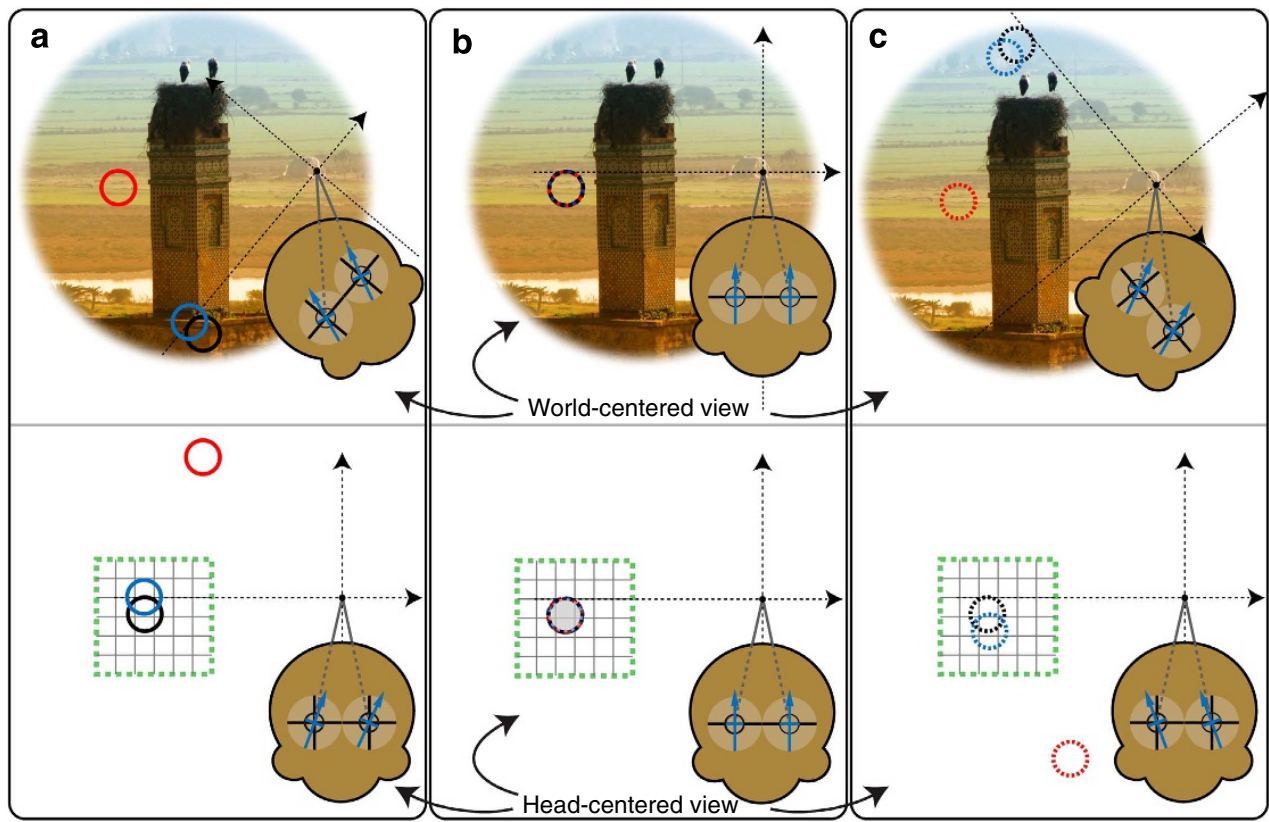

Figure 1 | Hypothetical reference systems used by V1 neurons to encode the visual world. The upper panel in $\mathbf{b}$ depicts a visual scene as seen by a monkey with the head upright. The (red-blue) dashed circle next to the tower delineates the schematic boundaries of a hypothetical V1 RF. The same $\mathrm{RF}$ is plotted in a head-centred representation of the visual field (lower panel). The blue arrows in the schematic monkey's head represent the orientation of the eyes relative to the head. Their vertical orientations indicate the absence of OC. a,c describe the oculomotor and visual consequences of roll tilting the head $50^{\circ} \mathrm{ccW}(\mathbf{a})$ or $\mathrm{CW}(\mathbf{c})$, eliciting OC. OC is indicated by the deviation of the blue arrows reflecting eye orientation from head vertical. The red circles (closed lines for ccw tilt, dashed for $\mathrm{cw}$ head tilt) reflect the position and boundaries of the area in the visual scene seen by the hypothetical $\mathrm{V} 1$ plotted relative to the head under the assumption of world-centred coding. The black circles represent the RF of the neuron, assuming a head-centred FOR and the blue circles the RF in an eye/retinal-centred FOR. The dotted green square (same as in Fig. 3) delineates the region in head centred stimulated to acquire a high resolution map of the RF.

typically preferred drifting gratings with bars parallel (perpendicular to the black line in Figs 3a-f and $4 \mathrm{~d}$ connecting the RF centre of mass to the fixation point) to the local vector lv (blue arrow in Fig. 4d) describing the rotation of a retina-centred RF. In this schematic representation (Fig. $4 \mathrm{~d}$ ), the rotation of the retinacentred RF was a consequence of a ccw OC induced by a $50^{\circ} \mathrm{cw}$ head tilt. On the other hand, neurons that were head-centred preferred drifting gratings with bars perpendicular (parallel to the black line connecting to RF centre of mass to the fixation) to $\mathbf{l v}$ (see Fig. 3a for an example). Finally, neurons whose orientation preference was roughly halfway between parallel and orthogonal (see Fig. $3 c$ for an example), exhibited a shift of their RF that only partially compensated for OC, leaving them in between a retina- and head-centred FOR. These examples suggest that the relationship between the orientation of the preferred gratings of a neuron and the orientation of lv determines the degree of RF shift in the head FOR. This is indeed the case as shown in Fig. 4e. In this figure, the degree of tilt-induced RF shift, in a head FOR, is captured by the ratio $\mathrm{SI}=\mathrm{RSa} / \mathrm{OCa}$ (RSa: angular RF shift relative to the head, black angle in Fig. 4d; OCa: ocular counter-roll angle, blue angle in Fig. 4d). This ratio varies between 0 , reflecting a $\mathrm{RF}$ that is fully head centred, and 1, for a RF that is retina centred. The polar plot of SI as function of the angular distance between the vertical on the orientation of the preferred grating (ov, brown double-head arrow in Fig. 4d) and lv, referred to as ODis (small green angle in Fig. 4d), depicted in Fig. 4e seems to follow a sine function. In other words, the magnitude of ODis, which varies between $0-90^{\circ}$, determines exactly how much a RF will shift relative to the head in response to tilt-induced OC. The maximum retinal shift is attained for an ODis of $0^{\circ}$, rendering the RF fully head centred. Conversely, an ODis of $90^{\circ}$ predicts that the RF will remain retina centred, thus shifted in a head FOR. We tested the assumption of a sinusoidal dependence by fitting a sine function to the data, and compared the explanation of the data provided by this fit with the one of a model assuming zero retinal RF shifts. The results obtained from this statistical comparison, which is presented in Fig. 6a (see the legend for details on the statistical parameters and data), are in full accordance with the hypothesis that the size of the shift of the RF location on the retina can be predicted by the sine rule described above.

As head tilts and the tilt-induced OC took place in complete darkness, head-centred neurons must rely on a non-visual signal $^{15,16}$ to shift their RF on the retina to compensate for OC. The distinctive visual feature of head-centred neurons that distinguishes them from the retina-centred ones is an orientation preference that would render them sensitive to OC-induced rotational motion of the visual background, provided a background were available. If this interpretation is correct, we would predict that direction selective neurons should shift their RF on the retina, thus not shifting in a head FOR, only for tiltinduced $\mathrm{OC}$ in a direction opposite to their preferred direction, leading to OC-induced background slip in their preferred direction. Conversely, they should not be head centred but retina centred, for OC in the same direction. This is actually the case, as demonstrated by the examples shown in Fig. 3 (compare the neuron depicted in Fig. 3d with the one in Fig. 3e; see Fig. $3 f$ for a neuron tested for both $\mathrm{cw}$ and $\mathrm{ccw}$ head roll tilts) and the results of a quantitative analysis based on 25 direction selective neurons summarized in Fig. 4f. This figure displays the relationship between the angular distance, referred to as DDis (large green angle in Fig. 4d) between the direction preference 
a
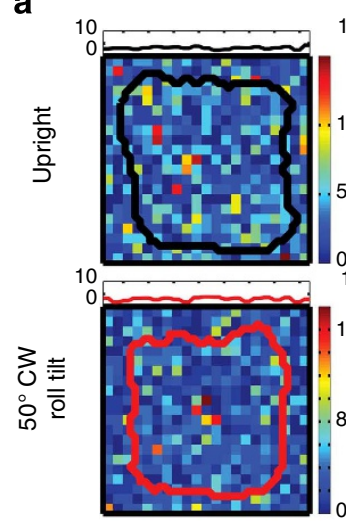
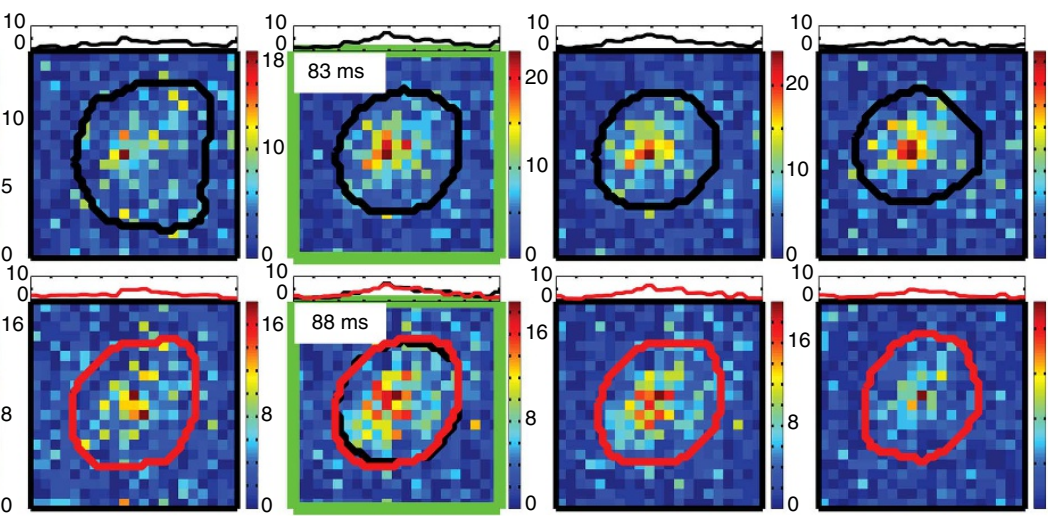

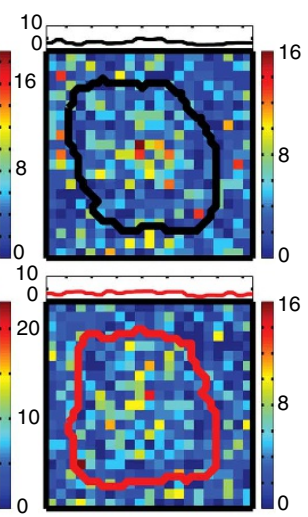

b

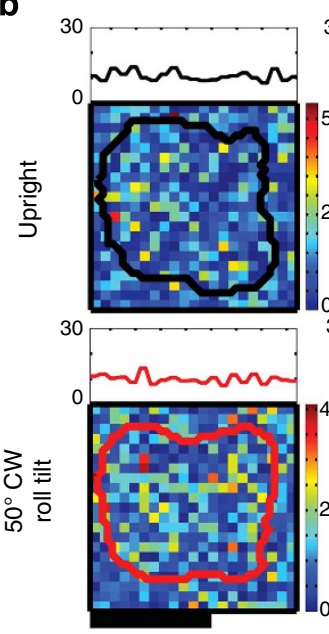

$1 \mathrm{~ms}$
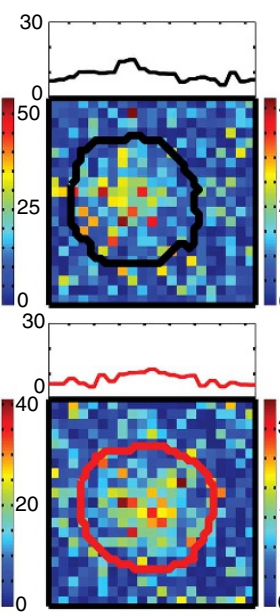

$50 \mathrm{~ms}$
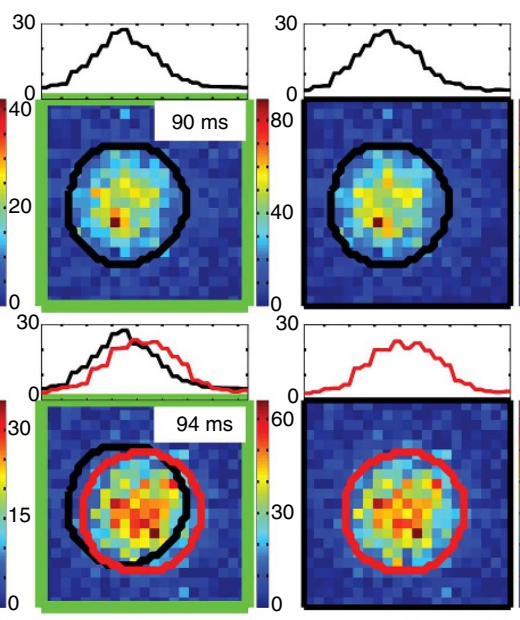

$100 \mathrm{~ms}$
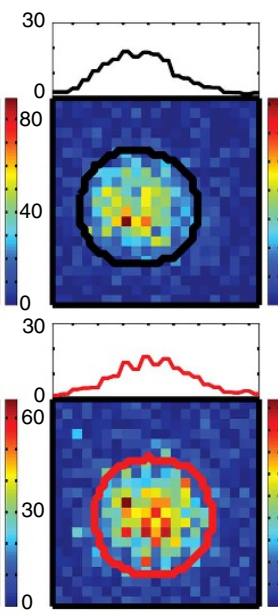

$150 \mathrm{~ms}$

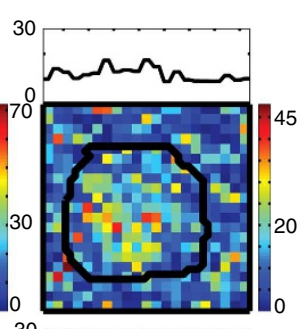

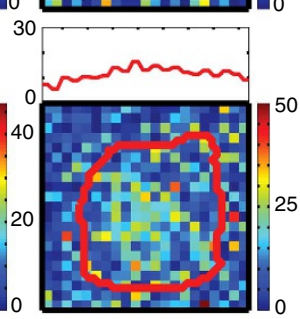

$200 \mathrm{~ms}$

Figure 2 | RFs of exemplary V1 neurons delineated by the reverse correlation. Spatial RF maps in a head-centred FOR obtained by using the reverse correlation technique for different points in time relative to stimulus onset ( $t=0 \mathrm{~ms}$ ). The upper rows in panels $\mathbf{a}$ and $\mathbf{b}$ show the RF maps of two neurons measured when the monkey was in the upright position. The lower row depicts the RF maps of the same neurons when the monkey was roll tilted $50^{\circ} \mathrm{cw}$. Both neurons had RFs located in the lower left part of the visual field. The maps highlighted by green frames depict 'peak maps'. These peak maps were obtained by evaluating the reverse correlation at the optimal temporal delay. The optimal temporal delay was determined as follows: maps were averaged along the $y$-dimension, yielding the curves plotted on the top of each map. The time delay yielding the curve with the largest peak was considered the optimal temporal delay. The black curves shown in addition to the red ones for head tilt replicate the curves shown in black for upright. The red or black contour lines demarcate the RF boundaries for the given condition. The black contour lines plotted on top of the red ones for $50^{\circ}$ roll tilt peak map duplicate the contour lines for the upright condition for comparative purposes. The colour bars gauge the overall number of actions potentials, elicited by the six stimulus repetitions, observed at a given time delay relative to the onset of the white probe stimulus, flashed at a specific position within the test field. Panel a depicts an exemplary head-centred neuron (RF location depicted by red circle in Fig. 4c), while panel b shows a retina-centred neuron (RF location depicted by blue circle in Fig. 4c). The horizontal scale bar corresponds to $1^{\circ}$ visual angle.

vector $\mathrm{dv}$ (brown arrow in Fig. $4 \mathrm{~d}$ ) and $\mathbf{l v}$ on the one hand and the amount of RF shift in a head FOR as captured by the ratio SI. We fitted a combination of sine and cosine to the direction selective neurons data to capture the influence of the sensitivity of a V1 neuron to a rotational retinal slip on the ratio SI and compared this fit with a simple retina coding model assuming an absence of RF shifts. The comparison of the two fits is shown in Fig. 6b. Only the former fit was significant, whereas the assumption of retinal coding could not fit the data (see Fig. 6 legend for details). Unlike neurons in layers 2 and 3, layer 4 neurons may actually be always retinocentric, independent of their specific orientation or direction preference. This is suggested by the analysis of a small and preliminary sample of $n=16$ layer 4 neurons (see Supplementary Fig. 5).

Effects of head tilt and OC on the orientation tuning curve. If a V1 neuron uses a non-visual signal (see discussion) reflecting the amount of OC to render its RF head centred, it might be expected to also keep its preferred orientation fixed relative to the head rather than keeping it fixed on the retina. If this were indeed the case, the preferred orientation on the retina should change by an amount corresponding to the amount of OC. Complete data sets that allowed us to compare the orientation or direction tuning for upright with the one for a least one of the two tilt conditions were available for a subgroup of 54 neurons from layers 2 and 3 (see Supplementary Fig. 6 for two examples). As shown in Fig. 4b, which plots the change of the orientation and direction preference in a head-centred FOR as function of the RF shift index SI, most head-centred neurons exhibited the expected change of their orientation preference on the retina, rendering their orientation preference head centred. Conversely, the neurons with retina-centred RFs also had retina-centred preferred orientations. Finally, the neurons with RFs intermediate between retina- and head-centred FORs also exhibited intermediate changes of their 
a

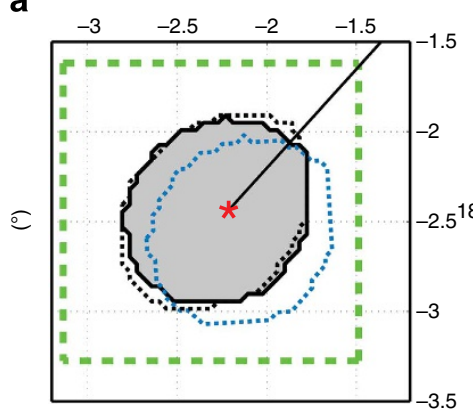

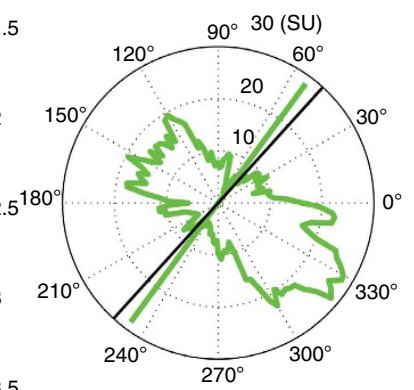

C

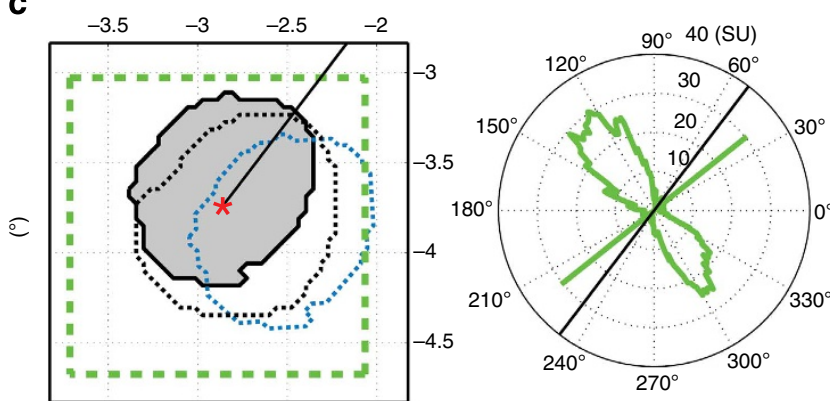

e

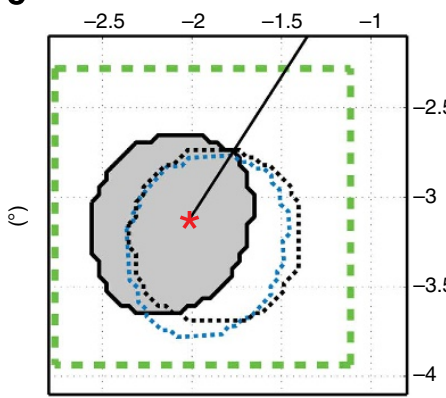

$\left({ }^{\circ}\right)$ b

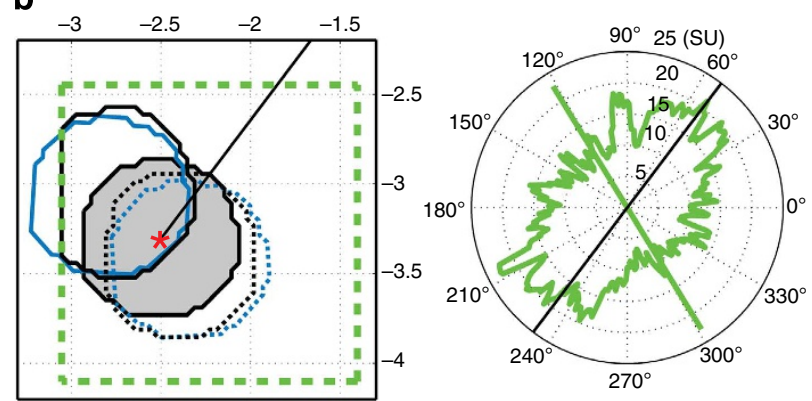

d

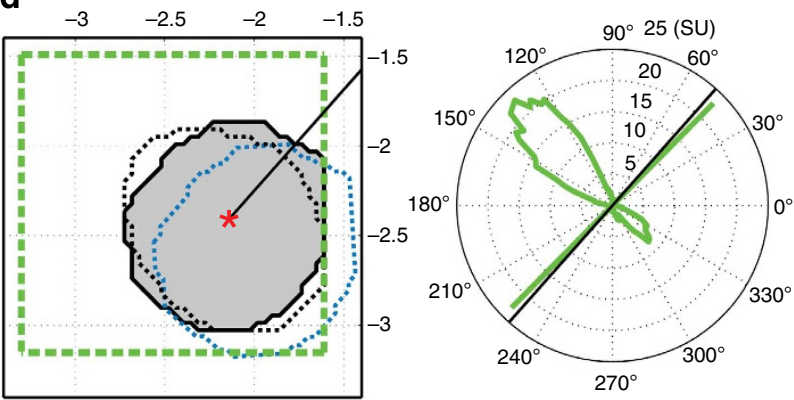

f
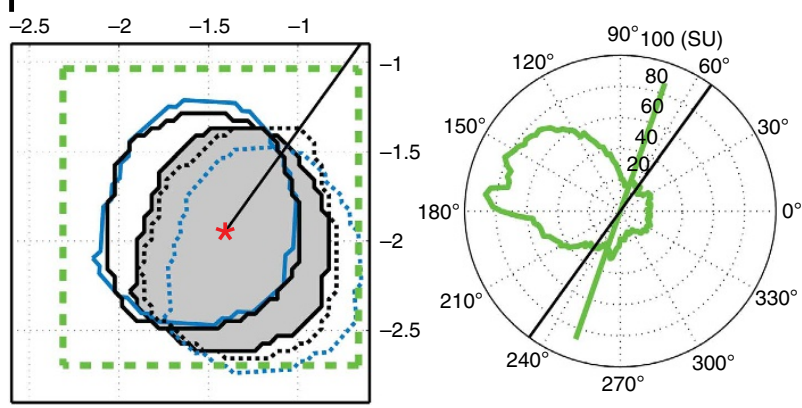

$\left({ }^{\circ}\right)$

Figure 3 | RFs and orientation/direction preference of exemplary V1 neurons. Each panel (a-f) shows the RF plot in a head-centred FOR (black square) for the upright position (gray area with black contour) superimposed by the RF map for the same neuron for the head roll tilted $50^{\circ} \mathrm{cW}$ (open area with black dotted contour) or/and $50^{\circ} \mathrm{ccw}$ (open area with black closed contour). In addition, hypothetical RF maps characterized by blue contour lines are shown. They are based on the assumption that RFs are retinocentric, independent of head orientation and the amount of OC induced by head tilts. These hypothetical RFs were obtained by rotating the RF map acquired for the upright condition by an amount corresponding to the OC of the eye contralateral to the ear downed by the head tilt. The rotation was performed around a line perpendicular to the monitor, passing through the fixation target. The straight black lines connect the centre of mass (red asterisk) of the RF map with the point of fixation. The dotted green square delineates the head-centred mapped area. The orientation/direction tuning curves shown next to the RF plots were obtained with the monkey upright, deploying a reverse correlation approach, which measured the number of spikes (SU) evoked by two repetitions of drifting grating of given orientation (chosen at random with a resolution of $3^{\circ}$ ) at the latency that had given rise to a 'peak map' when mapping RFs as explained in the legend of Fig. 2 . The straight green line superimposed on the tuning curve is parallel to the bars constituting the preferred grating. The straight black line superimposed on the tuning curve has the same slope as a line connecting centre of mass of the RF map with the point of fixation.

orientation preference as indicated by a correlation between the index SI and the amount of orientation/direction preference updating in a head FOR (see Fig. 4b).

\section{Discussion}

We show that the familiar notion of visual area V1 encoding the visual world as seen by the retina requires refinement. Actually, it is an artefact of experiments, in which head orientation is kept parallel to gravity. Yet, if the head is tilted, prompting a reflexive counter-rotation of the eyes, many neurons use non-visual information on head tilt-induced OC to shift their RF as well as their orientation preference on the retina, thereby at least partially compensating the rotation of the eyes relative to the head. The amount of compensation is predicted by the angle between two vectors. The first one describes the vertical on the preferred gratings of a neuron or-in the case of a direction selective neuron-its preferred drifting direction with the head upright. The second one describes how the OC would move a retinacentred RF across the retinal image of a world-centred background. This angle simply reflects the degree of sensitivity of the neuron to OC-induced background rotational motion, provided the head rotation took place in front of a world stationary visual background. In the case of an orientation selective neuron, the angular shift of RF position and orientation preference is predicted by the sine of this angular distance; in the case of a 
a

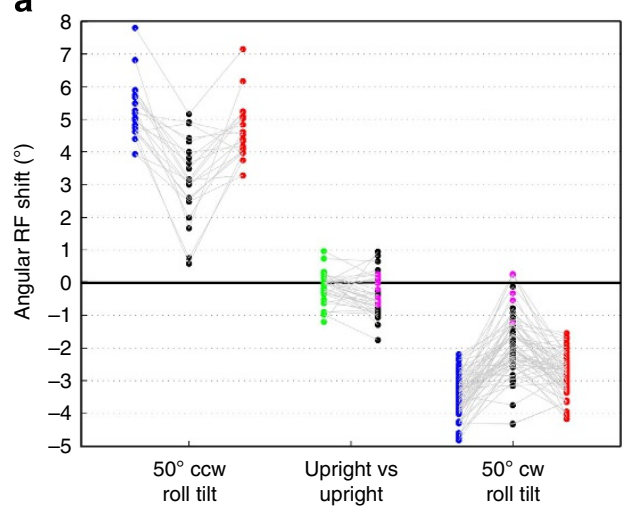

C

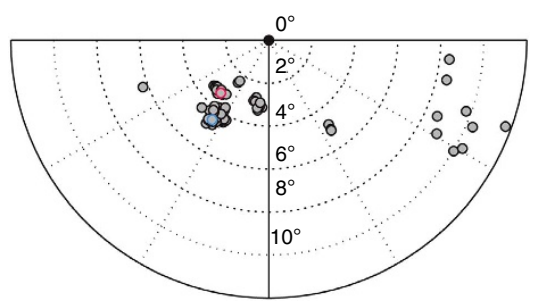

e

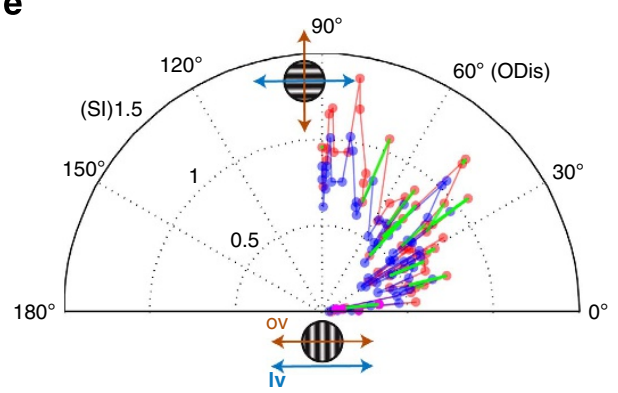

b

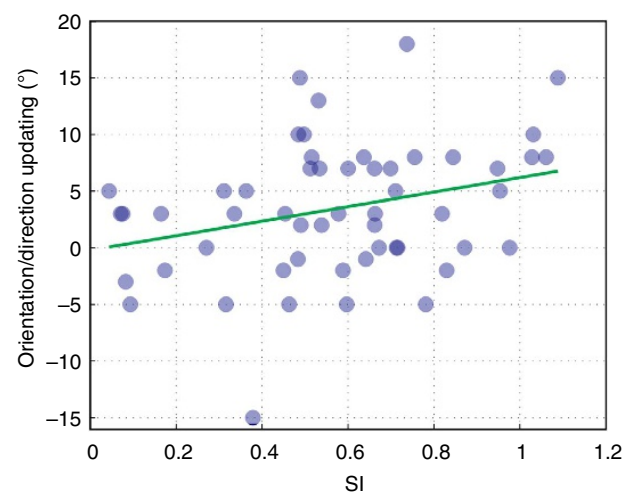

d

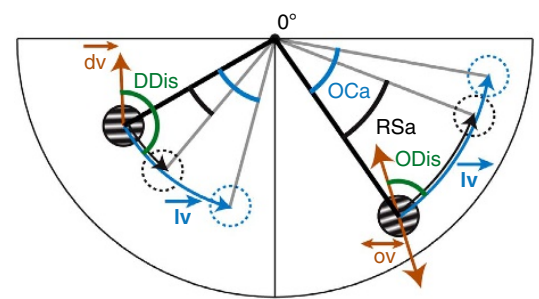

$\mathbf{f}$

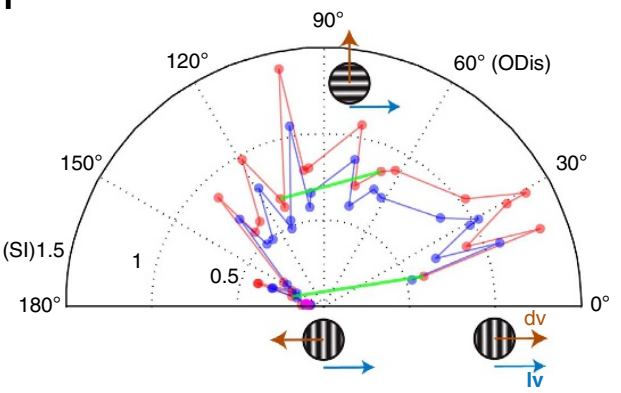

Figure 4 | Population response. (a) The black and magenta symbols specify the size of the angular RF shift of individual neurons ( $n=70)$ in a headcentred FOR relative to upright (first time upright), when the animal was tilted $50^{\circ} \mathrm{cw}, 50^{\circ} \mathrm{ccw}$ and back to upright (second time upright, coming from $50^{\circ}$ tilt). Magenta symbols denote nonsignificant RF displacements $(P<0.05$ using a bootstrap procedure, see Methods) and the black symbols significant ones. The seven neurons were tested in the upright and at least one roll tilt position. The red and blue symbols give the size of the associated tilt-induced $O C$ responses (see text for details). The green symbols characterize the OC of the right eye for upright. (b) Scatter plot of changes of orientation/direction preference in a head-centred FOR for 54 neurons from layers 2 and 3 versus RF shift indices SI (SI=RSa/OCa, see panel $\mathbf{d}$ for an explanation of the variables). Green line represents the weighted least-squares regression fit $(\beta=6.42, P<0.05)$. (c) RF positions in the visual field for upright (data from red and blue locations are shown in Fig. 2). (d) Illustrates (head centred view when tilted $50^{\circ} \mathrm{cW}$ ) the variables used to quantify the dependence of the RF shifts on the orientation/direction preferences of the neurons, presented in (e) and (f). The data presented in (e) and (f) are expressed in head-centred FOR. In (e) and (f) each data point represents data comparing responses of an individual neuron in the upright and a specific head tilt. (e) Summarizes the sample of 45 orientation selective neurons, (f) the 25 direction selective neurons. In these two polar plots the distance from the origin represents the ratio $\mathrm{SI}=\mathrm{RSa} / \mathrm{OCa}$, (RSa: angular $\mathrm{RF}$ shift relative to the head, OCa: ocular counter-roll angle; $\mathrm{SI}=0 \rightarrow$ head centred $\mathrm{RF}, \mathrm{SI}=1 \rightarrow$ retina centred $\mathrm{RF}$ ). In Fig. $4 \mathrm{e}$ the angular coordinate represents the variable ODis, in Fig. $4 \mathrm{f}$ the variable DDis. The blue and red data points represent the results when the amount of the OC of the eye contralateral or ipsilateral to the ear downed by the head tilt was considered as OC angle (OCa) when evaluating SI. The magenta symbols denote nonsignificant RF displacements. The green line connects two data points collected from the same neuron tested at two different head positions.

direction selective neuron, it is the combination of the sine and the cosine of this angle. For orientation selective neurons, if the two vectors are parallel, the retinal shift is fully compensatory, rendering RF and orientation preference head centred. If the two are orthogonal no shift results and the neuron remains retina centred. For the direction selective neurons, the preferred drifting direction also matters.

Our study suggests that the neuronal inventory of V1 contains two qualitatively different types of neurons, one representing the visual world relative to the retina and a second one relative to the head. Assuming $5^{\circ} \mathrm{OC}$, a head-centred neuron at an eccentricity of $20^{\circ}$ undergoes a tangential shift of its RF relative to the retina amounting to $2.67^{\circ}$. Accordingly, a head- and a retina-centred neuron seeing the same location in the visual field with the head upright would represent locations $2.67^{\circ}$ apart after OC. As retinaand head-centred neurons exhibit orthogonal orientation preferences, OC should shift elements of visual objects stimulating head-centred neurons by $2.67^{\circ}$ relative to elements accommodating the preferences of retina-centred neurons. In other words, OC would be expected to destroy the spatial continuity of the perceived object. However, our subjective experience suggests spatial continuity despite OC. Therefore, we must posit that also 


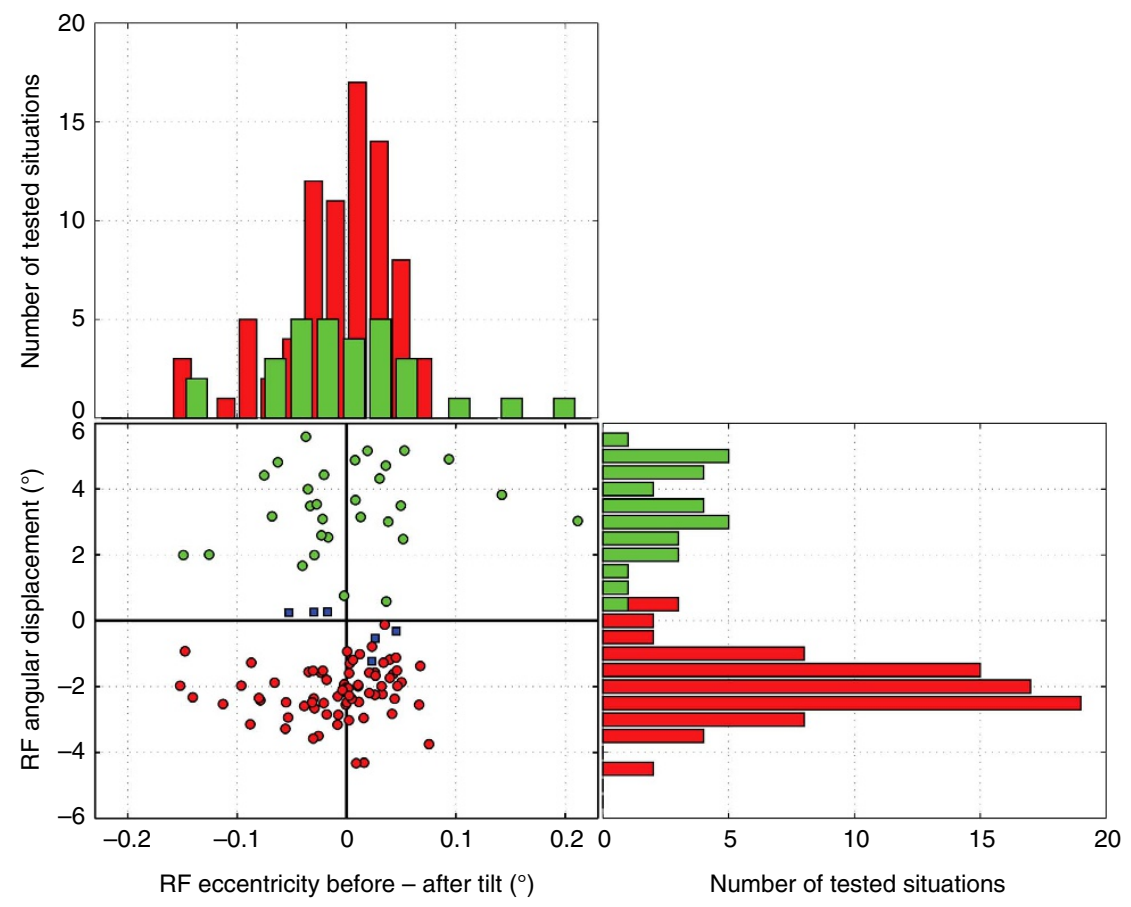

Figure 5 | RF displacements were purely angular. Angular shifts of individual RFs due to $50^{\circ} \mathrm{cw}$ (red) or ccw (green) head tilt (blue squares represent non-significant shifts) as function of changes in RF eccentricity. Note that the distribution of changes in RF eccentricity (upper histogram) is centred on zero irrespective of the head tilt direction $\left(n=79\right.$, mean $0.008^{\circ} \pm 0.049 \mathrm{~s}$.d. of the mean, $t$-test, $P=0.11$, for $50^{\circ} \mathrm{cw}$ head tilt; $n=30$, mean $0.007^{\circ} \pm 0.071 \mathrm{~s}$.d. of the mean, $t$-test, $P=0.9$, for $50^{\circ} \mathrm{ccw}$ head tilt) and irrespective of the amount of the tilt-induced RF shift (right side histogram). In other words RF displacements were purely angular.
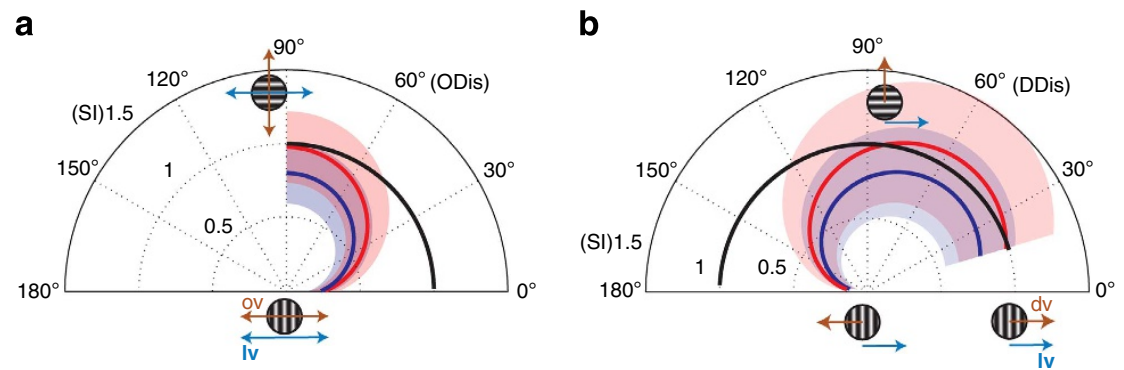

Figure 6 | Fitting the population response. (a) The blue and red curves depict fits of sinusoidal ( $A 1+A 2^{\star} \sin (O D i s)$ models to the data referring to the contralateral eye $\left(n=60, r^{2}=0.51\right.$, coefficients with $95 \%$ confidence bounds: $A 1=0.22(0.1,0.3), A 2=0.57(0.4,0.7), P>0.05$ Wilcoxon signed rank test applied to the residuals distribution) or the ipsilateral eye $\left(n=60, r^{2}=0.55\right.$, coefficients with $95 \%$ confidence bounds: $A 1=0.24(0.1,0.3)$,

$\mathrm{A} 2=0.72(0.55,0.9), P>0.05$ Wilcoxon signed rank test applied to the residuals distribution). The black curve depicts the behaviour of a model assuming that RF coordinates are retina centred (when applied to the actual data $r^{2}=-3.16, P<0.05$ Wilcoxon signed rank test applied to the residuals distribution, for the contralateral eye; $r^{2}=-1.0753, P<0.05$ Wilcoxon signed rank test applied to the residuals distribution, for the ipsilateral eye). (b) Shows the results for the direction selective neurons. In this case, the red and blue curves represent fits of the data based on a linear combination of a sine and a cosine function $\left(\mathrm{A} 1+\mathrm{A} 2^{*} \cos (\mathrm{DDis})+\mathrm{A} 3^{*} \sin (\mathrm{DDis})\right.$ to the data referring to the contralateral eye $\left(n=27, r^{2}=0.74\right.$, coefficients with $95 \%$ confidence bounds: $\mathrm{A} 1=0.4(0.2,0.5), \mathrm{A} 2=0.3(0.2,0.4), \mathrm{A} 3=0.35(0.1,0.5), P>0.05$ Wilcoxon signed rank test applied to the residuals distribution) or the ipsilateral eye $\left(n=27, r^{2}=0.71\right.$, coefficients with $95 \%$ confidence bounds: $\mathrm{A} 1=0.49(0.3,0.7), \mathrm{A} 2=0.37(0.2,0.5), \mathrm{A} 3=0.45(0.17,0.7), P>0.05$ Wilcoxon signed rank test applied to the residuals distribution). The black curve depicts the behaviour of a model assuming that RF coordinates are retina centred (when applied to the actual data $r^{2}=-2.02, P<0.05$ Sign-rank test applied to the residuals distribution, for the contralateral eye; $r^{2}=-0.57, P<0.05$ Wilcoxon signed rank test applied to the residuals distribution, for the ipsilateral eye). The shaded red and blue area corresponds to $90 \%$ confidence bounds.

neurons that were identified as retina centred in our experiments will prove to be head centred when tested under natural conditions. Hence, we may speculate that also retina-centred neurons are susceptible to a non-visual signal on eye rotation. Yet, to shift their RFs relative to the retina, this signal might require gating by concurrent visual activation of head-centred neurons, thereby unambiguously indicating that OC-induced retinal image slip took place. This coactivation was not available in our experiments as OC took place in darkness, and the RFs of individual neurons were probed with tiny non-oriented dot stimuli, precluding any relevant coactivation of neighbouring neurons. Yet, coactivation will arise during natural vision ${ }^{17}$. On the other hand, the non-visual signal on eye rotation must have privileged access to head-centred neurons, not contingent on 
concurrent visual activation. The reason is that only these neurons, characterized by radial orientation preferences, will be directly stimulated by OC-induced retinal image slip, entailing the need to distinguish this type of self-induced image slip from similar slip due to object motion in the world. We may add that the role of the non-visual signal on eye position that our findings suggest is very different from the one assumed by previous work $^{16}$. Durand and colleagues reported gain modulation of responses of V1 neurons by the Cartesian position of the eyes. According to the authors, this eye position sensitivity might enhance the visual processing of objects located straight ahead irrespective of gaze direction, in other words privileging a particular egocentric direction arguably of great ecological importance. V1 neurons have also been shown to exhibit changes in disparity coding with gaze direction changes ${ }^{18}$. However, none of these studies has provided any evidence for shifts of V1 RFs on the retina, precisely measured to maintain a stable head-centred position independent of OC and other types of torsional eye movements.

OC reduces the amount of tilt of the retinal image due to head tilt by roughly $10 \%$ in monkeys and humans. This reduction of image tilt is far too weak to fulfil the role that OC apparently plays in lateral-eyed animals: stabilizing the horizontal retinal meridian aligned with the horizon ${ }^{19,20}$. Actually, recent work on eye head coordination during voluntary gaze shifts ${ }^{9}$ suggests a different role of OC in primates, namely to increase the efficiency of these shifts. According to Crawford et al. ${ }^{9}$ (see also ${ }^{21,22}$ ) a new eye head gaze shift following a preceding one involving a head tilt in a particular direction will be more likely to require a gaze torsion in the opposite direction. As the eyes will already be counter-rolled in this direction, the eyes will have a head start. Work on the neural underpinning of the OC is in accordance with the assumed role in optimizing saccades as part of upcoming eye head shifts. It suggests that OC is a consequence of neural integration of otolith-derived signals by the oculomotor integrator serving the saccadic system ${ }^{9}$. The benefit of the OC to facilitate the fast and efficient repositioning of gaze is associated with the need to take OC into account when interpreting the orientation of retinal images. Our findings suggest how V1 may use non-visual information on OC to filter out the visual consequences of OC, an important first step towards reconstructing a veridical interpretation of the orientation of the external visual world. It is an intriguing speculation that the non-visual signal exploited by V1 may actually be the output of the oculomotor integrator, that is, the same signal that is responsible for OC to occur in the first place.

\footnotetext{
Methods

Subjects and single-unit recording. All the animal procedures complied with the NIH guide for the care and use of laboratory ${ }^{23}$ animals and were approved by the local animal care committee (Regierungspraesidium Tüebingen, FG Tierschutz). Two male rhesus monkeys (Macaca mulatta) M1 (8 years old) and M2 (9 years old) participated in this study. Both monkeys were implanted with head posts and 2Dsearch coils allowing the recording of horizontal and vertical eye position. In both monkeys, torsion was measured by comparing video images of the eyes acquired when the monkeys were fixating with the head upright and when they were roll tilted. During spike recording sessions, only the right eye torsion was measured, as preceding binocular measurements on the two monkeys used had allowed us to establish that the very small differences $\left(<0.8^{\circ}\right)$ of the OC responses of the two eyes followed a simple and reliable regularity (see ${ }^{12}$ for details). Extracellular action potentials were recorded with commercial glass-coated microelectrodes (impedance at $1 \mathrm{kHz}: 0.5-1 \mathrm{MOhm}$ ). The electrode was inserted through the intact dura and advanced very slowly $\left(1 \mathrm{~mm} \mathrm{~h}^{-1}\right)$ to give the tissue sufficient time to relax from the pressure exerted by the electrode. Electrode depth relative to first visually evoked activity, the level of spontaneous activity, spike morphology, the preponderance of orientation versus direction selectivity and other criteria described by Snodderly and Gur ${ }^{13}$ were used to identify the layer of a recorded unit. Spikes of well-isolated single units (see Supplementary Fig. 7 and Supplementary Fig. 8) were discriminated online by real-time sorting software based on template matching (Alpha Omega Engineering).
}

RF mapping procedures. Testing individual units involved acquiring a high resolution map of the $\mathrm{RF}$ with the monkey fixating a tiny dot presented straight ahead on a monitor at $50 \mathrm{~cm}$ distance (dot size $0.08^{\circ}, 0.75^{\circ}$ fixation window) in the upright position by flashing a white probe $\left(0.08^{\circ}\right.$ for most of the sessions, in a minority of cases $0.12^{\circ}, 50 \mathrm{~ms}$ duration followed by $50 \mathrm{~ms}$ black screen) on a black background. Next, orientation tuning curves were collected using drifting gratings ( $400 \mathrm{~ms}$ duration followed by $100 \mathrm{~ms}$ black screen), which changed orientation in steps of $3^{\circ}$. Subsequently, the monkey was roll tilted, together with the monitor, $50^{\circ}$ $\mathrm{cw}$ or ccw. Once the roll tilt position was reached and OC had plateaued (within less than $10 \mathrm{~s}$ ), we remapped the same head-centred region to acquire the RF map. Furthermore, we acquired photographs of the right eye, while fixating, to measure the amount of OC relative to eye photographs obtained for upright. For some cells, we re-run the orientation tuning curves. If the cell was not lost, we brought the monkey with the monitor back to the upright position, to repeat the RF mapping for upright. Only in a few cases was it possible to also analyse the unit for the opposite head position.

Stability of recordings and visual stimulation. To reveal the small receptive shifts reported, we had to resort to extremely strict criteria when isolating and selecting neurons, ensuring that we would map the RF of one and the same single unit, tested under different head tilt conditions, without any interference from other units. Actually, the need to tilt the monkey's body and head together often resulted in a loss of proper isolation, probably because of subliminal vibrations associated with the acquisition of the new orientation and/or movement of the brain relative to the electrode because of changes of the head and body orientation relative to gravity. This is why we could obtain single units with well-isolated stable spikes allowing the reliable mapping of their RF in the upright and when the head was roll tilted in only about $10 \%$ of the recording sessions (see also Supplementary Note 1-3).

As tilting the monkey and the monitor relative to the gravitational vector will induce torques for components away from the axis of rotation, one might be worried about the possibility of small relative movements between the monkey's head and the monitor. To check if such relative displacements indeed occurred, we attached an infrared camera to the monkey's head holder to image two small infrared light sources located on the upper and lower edges of the monitor. Before roll tilting the monkey and the monitor together, the positions of these monitor infrared light sources were acquired with the camera and reacquired ones the monkey had reached the final roll tilt position. If any change between the two sets of infrared light sources coordinates (upright versus roll tilt) was detected, the difference was computed (we observed a maximal shift of $0.2^{\circ}$ along the horizontal axis of the monitor and a maximal shift of $0.06^{\circ}$ along the vertical one) and used to correct both, the position of the fixation target on the screen and the coordinate of the monitor portion used for the RF mapping procedure (dotted green square in Fig. 1), thus preserving a constant spatial relation between the fixation target and the location of the monitor mapped. In this way, we ensured that the monkey was looking all the time straight ahead and we mapped the same head-centric location of the visual field, independent of the orientation of the monkey and the monitor.

Measurement of ocular counter-roll. Ocular counter-roll (OC) was calculated by identifying clearly visible iris landmarks (see Supplementary Fig. 9 for an example). Next, a line was drawn between a distinct part of the landmark and the centre of the pupil. By subtracting the orientation of the line for the upright orientation (Supplementary Fig. 9a) of the head from the orientation of the line for the head roll tilted (Supplementary Fig. 9b), we could calculate the amount of OC induced by roll tilting the head.

To quantify the reliability of using visual landmarks to assess the torsional state of the eye, we adopted the procedure described before on a series of individual snapshots of the eye with the monkey being upright and compared the individual eye torsion values with the mean eye torsion (defined as $0^{\circ}$ ) for a typical experiment. Hence, assuming reliable landmark choices and the absence of residual miniature torsional eye movement, all the torsion values should correspond to $0^{\circ}$. Supplementary Table 2 summarizes the results for the two monkeys. The resulting distributions were centred on zero with very small standard deviations.

Reconstruction of binocular ocular counter-roll. During the neuronal recording sessions only the right eye torsion was measured. We deduced the left eye OC, based on separate sessions in which binocular OC was measured. In the binocular sessions, we assessed the mean difference of the OC between the two eyes $\left(<0.8^{\circ}\right.$, see ref. 12 for details) and we used this difference to deduce the amount of the OC of the left eye by adding (when cw head tilt was performed) or subtracting (when $\mathrm{ccw}$ head tilt was performed) this number from the measured right eye OC.

Calculation of the orientation/direction preference. To determine the orien tation/direction preference of a neuron, we first classified each neuron as orientation or direction selective based on its responses to drifting gratings resorting to the approach described by Wörgötter and $\mathrm{Eysel}^{24}$. In brief, we applied a Fast Fourier Transformation (FFT) to the polar plot (the latter resulting from reverse correlating the spikes with the grating's orientation). The zero order gain component of the resulting spectrum corresponds to the spontaneous activity, 
the first order component is interpreted as the strength of the direction selectivity and the second order component as the strength of the orientation selectivity (the phase of the directional and orientational components were not considered as we were only interested in the gain of each component). A neuron was counted as orientation selective but not directional if the gain of the orientation component was greater than the gain of the direction component. Conversely, it was considered direction selective if the gain of the direction component was greater than the gain of the orientation component. If the neuron was classified as direction selective, we applied a Rayleigh test to assess the significance of the mean direction using the original polar plot. In case the neuron was classified as orientation selective we considered the two lobes of the polar plot separately and applied Rayleigh tests on both to assess the significance of the circular mean for each of them. The preferred orientation axis was given by the average of the two means, each one rotated by $90^{\circ}$. The calculation of tilt-dependent orientation/ direction updating is described in the legend to Supplementary Fig. 6.

Determination of the RF boundaries for visualization proposes. In Figs 2 and 3 the contour lines representing the RF boundaries for the different conditions are drawn for visualization purposes only. We obtained those contours by applying the Otsu's method ${ }^{25}$ for global image thresholding to the maps resulting from the reverse correlation procedure, yielding binary maps. Next, we applied the Matlab function bwboundaries. $m$ to the binary maps. This function traces the exterior boundaries of the RF by detecting boundary pixels belonging to the same RF contour.

Statistical analysis of RF shifts. To assess the statistical significance of the RF shift, we applied a bootstrap method. We estimated the variability of the RF measurement by simulating 1,000 repetitions of the measurement based on the following considerations: to map the RF using reverse correlation, each location of the grid parsing the part of the visual field containing the RF (see Fig. 1) was stimulated by a squared $\left(0.08 \times 0.08^{\circ}\right)$ white probe six times, providing a RF map based on six repetitions. As a start of the bootstrap procedure, we derived six RFs maps, based on one single repetition each. Each of these RF maps was smoothed by applying a 2D spatial filter (circular averaging filter with 5 pixels radius). Next, we constructed 1,000 new RF maps based on the six single-trial RFs maps as follows: first, a pixel in one of the 1,000 constructed RF maps was chosen randomly (with replacement) from the corresponding pixel of the six single-trial RF maps. This was done for each pixel in the map and 1,000 times to obtain 1,000 RF maps for the upright condition. Then the same procedure was repeated for the roll tilt condition. Next, we performed normalized 2D cross-correlations between the 1,000 RF maps for upright and the 1,000 RF maps for a roll tilt condition. Then, we extracted the spatial coordinates associated with the maximum cross correlation coefficient. Note that we deployed a standard cartesian $2 \mathrm{D}$ cross correlation rather than a $2 \mathrm{D}$ correlation in a polar coordinate system. This seems allowed as the two methods will yield the same location of the peak cross correlation coefficient because of the symmetric nature of the RF maps. The cross correlation gave two distributions (each containing 1,000 values) for the $x$ - and $y$-coordinates of RF locations leading to maximum cross-correlations. The means of these two distributions describe how much one should displace the RF obtained in the roll tilt position over the RF obtained in the upright position to obtain a maximum cross correlation. We determined the $95 \%$ range of the $x$ - and $y$ - distributions (by determining the $2.5 \%$ left most and 2.5\% right most from the centre of the 1,000 simulation). When the $95 \%$ of one of the distributions did not overlap with 0 , the displacement was considered as statistically significant. This procedure corresponds to a two-tailed test with a significance level of $P=0.05$. The s.d. of these two distributions are shown in Supplementary Fig. 3 panels c,d for all the tested neurons. The means of these two distributions are taken as the actual RF displacement.

\section{References}

1. Hubel, D. H. \& Wiesel, T. N. Receptive fields of single neurones on the cat's striate cortex. J. Physiol. 148, 574-591 (1959).

2. Von Helmholtz, H. Handbuch der physiologischen Optik. Vol. 3 (Leipzig: Leopold Voss, 1867).

3. Von Holst, E. \& Mittelstaedt, H. Das Reafferenzprinzip. Naturwissenschaften 37, 464-476 (1950)

4. Haarmeier, T., Thier, P., Repnow, M. \& Petersen, D. False perception of motion in a patient who cannot compensate for eye movements. Nature 389, 849-852 (1997).

5. Denney, D. \& Adorjani, C. Orientation specificity of visual cortical neurons after head tilt. Exp. Brain. Res. 14, 312-317 (1972).

6. Kingma, H., Stegeman, P. \& Vogel, R. Ocular torsion induced by static and dynamic visual stimulation and static whole body roll. Eur. Arch. Otorhinolaryngol. Suppl 1, s61-s63 (1997).
7. Collewijn, H., Van der Steen, J., Ferman, L. \& Jasen, T. C. Human ocular counterroll: assessment of static and dynamic properties from electromagnetic sclera coil recordings. Exp. Brain Res. 59, 185-196 (1985).

8. Linwong, M. \& Herman, S. J. Cycloduction of the eyes with head tilt. Arch Ophthamol. 85, 570-573 (1971).

9. Crawford, J. D., Tweed, D. B. \& Vilis, T. Static ocular counterroll is implemented through the 3-D integrator. J. Neurophysiol. 90, 2777-2784 (2003).

10. Gibson, J. J. \& Mowrer, O. H. Determinants of the perceived vertical and horizontal. Psychol. Rev. 45, 300-323 (1938).

11. Judge, S. J., Richmond, B. J. \& Chu, F. C. Implantation of magnetic search coils for measurement of eye position: an improved method. Vision. Res. 20, 535-538 (1980).

12. Daddaoua, N., Dicke, P. W. \& Thier, P. Non-human primates exhibit disconjugate ocular counterroll to head roll tilts. Vision. Res. 51, 1986-1993 (2011).

13. Snodderly, D. M. \& Gur, M. Organization of striate cortex of alert, trained monkeys (Macaca fascicularis): Ongoing activity, stimulus selectivity, and widths of receptive field activation regions. J. Neurophysiol. 74, 2100-2125 (1995).

14. Jones, J. P. \& Palmer, L. A. The two-dimensional spatial structure of simple receptive fields in cat striate cortex. J. Neurophysiol. 58, 1187-1211 (1987).

15. Buisseret, P. \& Maffei, L. Extraocular proprioceptive projections to the visual cortex. Exp. Brain Res. 28, 421-425 (1977).

16. Durand, J. B., Trotter, Y. \& Celebrini, S. Priviliged Processing of the StraightAhead Direction in Primate Area V1. Neuron. 66, 126-137 (2010).

17. Das, A. \& Gilbert, C. D. Topography of contextual modulations mediated by short-range interactions in primary visual cortex. Nature 399, 655-661 (1999)

18. Trotter, Y. \& Celebrini, S. Gaze direction controls responses gain in primary visual-cortex neurons. Nature 389, 239-242 (1999).

19. Baarsma, E. A. \& Collewijn, H. Eye movements due to linear acceleration in the rabbit. J. Physiol. 245, 227-247 (1975).

20. Dickman, J. D. \& Angelaki, D. E. Three-dimensional organization of vestibular related eye movements during off-vertical axis rotation and linear translation in pigeons. Exp. Brain Res. 129, 391-400 (1999).

21. Klier, E. M., Wang, H. \& Crawford, J. D. Three-dimensional eye-head coordination is implemented downstream from the superior colliculus. J. Neurophysiol. 89, 2839-2853 (2003).

22. Monteon, J. A., Constantin, A. G., Hongying, W., Martinez-Trujillo, J. \& Douglas Crawford, J. Electrical stimulation of the frontal eye fields in the headfree macaque evokes kinematically normal 3D gaze shifts. J. Neurophysiol. 104, 3462-3475 (2010).

23. Thier, P. \& Erickson, R. G. Responses of visual-tracking neurons from cortical area MST-I to visual, eye and head motion. Eur. J. Neurosci. 4, 539-553 (1992).

24. Wörgötter, F. \& Eysel, U. T. Quantitative determination of orientational and directional components in the response of visual cortical cells to moving stimuli. Biol. Cybern. 57, 349-355 (1987).

25. Otsu, N. A Threshold Selection Method from Gray-Level Histograms. IEEE. Trans. Syst. Man. Cybern. 9, 62-66 (1979).

\section{Acknowledgements}

This study is part of the research programme of the Bernstein Center for Computational Neuroscience, Tübingen, funded by the German Federal Ministry of Education and Research (BMBF FKZ: 01GQ1002, TP C3).

\section{Author contributions}

N.D., P.W.D. and P.T. designed the experiment; N.D. performed the experiment; N.D. and P.W.D. analysed the data; N.D. and P.T. wrote the paper.

\section{Additional information}

Supplementary Information accompanies this paper at http://www.nature.com/ naturecommunications

Competing financial interests. The authors declare no competing financial interests.

Reprints and permissions information is available online at http://npg.nature.com/ reprintsandpermissions/

How to cite this article: Daddaoua, N. et al. Eye position information is used to compensate the consequences of ocular torsion on V1 receptive fields. Nat. Commun. 5:3047 doi: 10.1038/ncomms4047 (2014). 